\title{
The antioxidant role of Sideritis caesarea infusion against TCA toxicity in rats
}

\author{
Ismail Celik* and Mehmet Salih Kaya \\ Department of Biology, Faculty of Arts and Sciences, Yuzuncu Yil University, 65080 Van, Turkey
}

(Received 30 June 2010 - Revised 18 August 2010 - Accepted 20 September 2010 - First published online 16 November 2010)

\section{Abstract}

Sideritis caesarea (SC) Duman, Aytac\&Baser is a member of the Lamiaceae family. The present study was designed to investigate the antioxidant properties of the aerial parts of island green tea SC against TCA effects in rats. Biomarkers selected for monitoring antioxidant capacity were the activities of glutathione reductase (GR), superoxide dismutase (SOD), glutathione-S-transferase, catalase (CAT), GSH level and malondialdehyde (MDA) content in various organs of rats. Three experimental groups, A (untreated = control), B (only TCA-treated) and C (TCA + SC-treated), were studied. At the end of the $50 \mathrm{~d}$ experiment, the MDA content in tissues increased significantly in group B, whereas no significant changes were observed in group C as compared with that of the control group. Antioxidant enzyme activities such as SOD and CAT increased significantly in the brain, liver and kidneys of group B but decreased significantly in group C as compared with group B. The GSH level and GR activity increased significantly in the brain and liver of group C as compared with the control and TCA-exposed rats. Hence, the study reveals that the constituents present in SC impart protection against chemical-induced oxidative injury that may result in the development of cancer.

Key words: Sideritis caesarea (Lamiaceae): Medicinal food: TCA: Antioxidant defence systems: Malondialdehyde: Rats

Today's world is increasingly seeking ways to replace synthetic drugs with the therapeutic power of natural products. Traditional folk medicine had already found the secret of healing in nature. Medicinal plants have been used for therapeutic purposes since the beginning of civilisation. Following a recent period in Western medicine when plant medicines were shunned, there has been a resurgence of interest in plant compounds with beneficial pharmacological properties ${ }^{(1)}$. Generally, Sideritis spp., including Sideritis caesarea (SC), are widely used in the folk medicine, especially for inflammatory, antiulcerogenic, anticandidal, and alimentary diseases and gastrointestinal disturbances ${ }^{(2-4)}$. Their effectiveness has been attributed mainly to their antioxidant properties originating from terpenoids and flavonoids in their composition ${ }^{(5)}$.

Reactive oxygen species such as the superoxide anion $\left(\cdot \mathrm{O}_{2}^{-}\right)$, hydroxyl radical $(\cdot \mathrm{OH})$, hydroxyl ion $\left(\mathrm{OH}^{-}\right)$and $\mathrm{H}_{2} \mathrm{O}_{2}$ are known to play a major role in either the initiation or the progression of carcinogenesis by inducing oxidative stress ${ }^{(6)}$. Peroxides and $\cdot \mathrm{O}_{2}^{-}$produce cytotoxicity/genotoxicity in the cellular system ${ }^{(7,8)}$. A large number of studies have supported the hypothesis that oxidative damage to
DNA, lipids and proteins may contribute to the development of CVD, cancer and neurodegenerative diseases. Reactive oxygen and nitrogen species are formed in the human body, and endogenous antioxidant defences are not always sufficient to counteract them completely. Dietderived antioxidants may therefore be particularly important in protecting against chronic diseases ${ }^{(9-13)}$. Hopefully, the genotoxic effects of toxicants can be minimised by the modulation of physiological detoxification processes. Many naturally occurring compounds with antioxidative action are now known to protect cellular components from oxidative damage and to prevent diseases. In addition, many natural compounds are now known to have a modulator role on physiological functions and biotransformation reactions involved in the detoxification process, thereby affording protection against cytotoxic, genotoxic and metabolic actions of environmental toxicants ${ }^{(14)}$.

Despite the reasons mentioned above, little is known regarding the antioxidant capacity of SC, popular as a herbal tea in Turkey. In order to gain a rational understanding of the benefits of SC, it is necessary to clarify comprehensively the mechanisms of antioxidant capacity

Abbreviations: CAT, catalase; GR, glutathione reductase; GST, glutathione-S-transferase; MDA, malondialdehyde; SC, Sideritis caesarea; SOD, superoxide dismutase.

*Corresponding author: I. Celik, fax +90 432 2251114, email icelik_65@yahoo.com 
responsible for the improving effects of the plant. Also, in the present study, our attention is focused on the preventive actions of this natural product, as these properties are known to be closely related to the prevention and therapy of cancer. To achieve this, SC infusion treatment was given orally in drinking water because the effect of plants represents a well-characterised mechanism in nutrition and because SC is widely used as a beverage by humans in Turkey. The antioxidant capacity of crude and pure products of SC on some phase II detoxification enzymes and lipid peroxidation was evaluated during the exposure to TCA in rats.

\section{Materials and methods}

\section{Chemicals}

Thiobarbituric acid, butylated hydroxytoluene, TCA, EDTA, GSH, metaphosphoric acid, 5,5'-dithiobis-(2-nitrobenzoic acid), trihydroxymethyl aminomethane (Tris), 1-chloro2,4-dinitrobenzene, GSSG, $\beta$-NADPH, potassium dihydrogen phosphate $\left(\mathrm{KH}_{2} \mathrm{PO}_{4}\right), \mathrm{H}_{2} \mathrm{O}_{2}$ and $\mathrm{NaCl}$ of technical grade used in the present study were supplied by Sigma Chemical Company (St Louis, MO, USA). Kits for the analysis of antioxidant enzymes were supplied by Randox Laboratories Limited (Crumlin, UK). Authenticated dry plant material of SC was purchased from a local herb store.

\section{Animals}

Sprague-Dawley rats, weighing 150-200g and averaging 4 months old, were provided by the animal unit of the Medical School of Yuzuncu Yil University and were housed in three groups, each group containing six rats. The animals were housed at $20 \pm 2^{\circ} \mathrm{C}$ in a $12 \mathrm{~h}$ light $-12 \mathrm{~h}$ dark cycle. All animals were fed a wheat-soyabean meal-based diet and water ad libitum in stainless steel cages and received humane care according to the criteria outlined in the 'Guide for the Care and Use of Laboratory Animals' prepared by the National Academy of Science and published by the National Institutes of Health ${ }^{(15)}$. The ethical regulations were followed in accordance with the national and institutional guidelines for the protection of animal welfare during the experiments. Also, the present study was approved by the Ethic Committee of the Medical School of Yuzuncu Yil University.

\section{Preparation of plant infusion and treatment}

Authenticated dry plant (Dağ ada çayı) was purchased from a local herb store. A voucher specimen has been deposited at the Department of Biology, Faculty of Sciences and Arts, Yuzuncu Yil University, Van, Turkey, for future reference. Plant infusion was prepared by soaking $2.5 \mathrm{~g}$ of the aerial parts of the dry plant in 1 litre of boiled water for $5 \mathrm{~min}$ to simulate actual brewing conditions for tea consumed by human adults. The dose of TCA was selected on the basis of a $0 \cdot 2 \%$ (approximately $0 \cdot 012 \mathrm{M}$ ) concentration at which carcinogen was produced in vivo, as drinking water was administered orally to rats ad libitum for $50 \mathrm{~d}$ continuously ${ }^{(16)}$. While the TCAexposed rats were administered the TCA solution orally as drinking water, the treatment groups with TCA + plant infusions received TCA + SC infusion orally with selected doses of aqueous infusions. The control group was fed boiled and cooled water only. Treatments were initiated synchronistically.

\section{Preparation of tissue supernatant and erythrocyte homogenate}

At the end of the treatments, blood samples were obtained by cardiac puncture using a syringe for the determination of biochemical constituents. For this purpose, the blood samples were put immediately into Si disposable glass tubes with EDTA as an anticoagulant. The blood samples were centrifuged at $4000 \mathrm{~g}$ for $15 \mathrm{~min}$ at $4^{\circ} \mathrm{C}$, and erythrocyte pellets were obtained. Then, the pellets were washed three times with physiological saline (0.9\% $\mathrm{NaCl}$ ). The $\mathrm{GSH}$ concentration in erythrocytes and tissues was measured immediately after the animals were killed because of the tremendous loss of GSH. The GSH level, malondialdehyde (MDA) content and the activities of superoxide dismutase (SOD), glutathione reductase (GR), catalase (CAT) and glutathione-S-transferase (GST) in erythrocytes were measured in the pellets.

The tissues were dissected and placed in Petri dishes. After washing the tissues with physiological saline $(0.9 \%$ $\mathrm{NaCl}$ ), samples were taken and maintained at $-78^{\circ} \mathrm{C}$ until analysis. The tissues were homogenised for $5 \mathrm{~min}$ in a $50 \mathrm{~mm}$-ice-cold $\mathrm{KH}_{2} \mathrm{PO}_{4}$ solution (1:5, w/v) using a glass-porcelain homogeniser $(20 \mathrm{kHz}$ frequency ultrasonic; Jencons Scientific Company, Bridgeville, PA, USA) for $5 \mathrm{~min}$ and then centrifuged at $7000 \mathrm{~g}$ for $15 \mathrm{~min}$. All processes were carried out at $4^{\circ} \mathrm{C}$. Supernatants and haemolysate were used to determine antioxidant defence systems and MDA concentration.

\section{Biochemical analysis}

The MDA concentration in erythrocytes and tissues was determined using the method described by Jain et al. ${ }^{(17)}$ based on thiobarbituric acid reactivity. Briefly, $0.2 \mathrm{ml}$ of erythrocyte pellets or supernatant obtained from tissues, $0.8 \mathrm{ml}$ of phosphate buffer ( $\mathrm{pH} 7 \cdot 4$ ), $0.025 \mathrm{ml}$ of butylated hydroxytoluene and $0.5 \mathrm{ml}$ of $30 \%$ TCA were added to the tubes and mixed. After $2 \mathrm{~h}$ incubation at $-20^{\circ} \mathrm{C}$, the mixture was centrifuged $(400 \mathrm{~g}$ ) for $15 \mathrm{~min}$. Then, $1 \mathrm{ml}$ of the supernatant was taken and added to each tube, followed by the addition of $0.075 \mathrm{ml}$ EDTA $(0.1 \mathrm{M})$ and $0.25 \mathrm{ml}$ of $1 \%$ thiobarbituric acid. These tubes with Teflon-lined screw caps were incubated at $90^{\circ} \mathrm{C}$ in a 


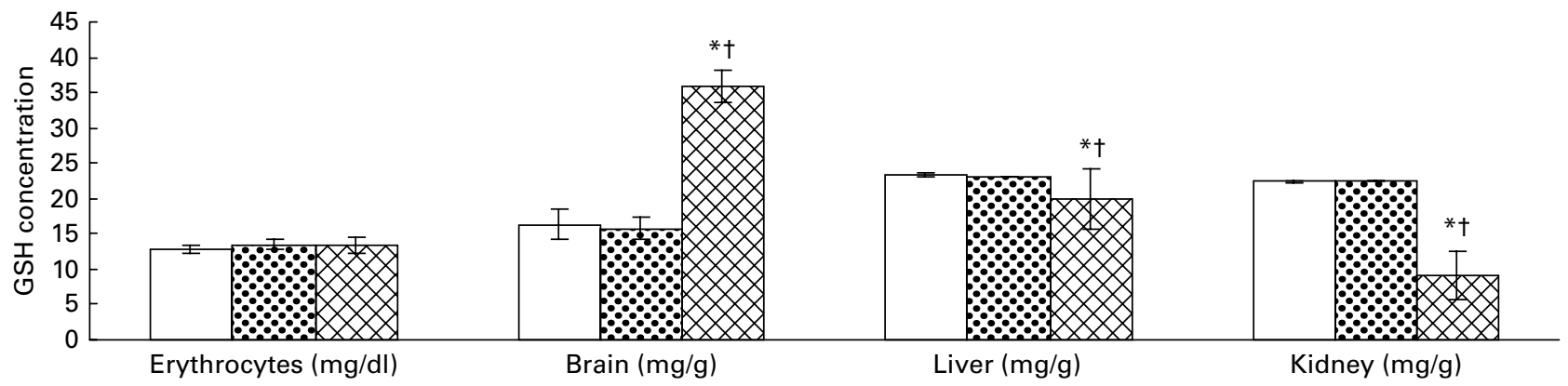

Fig. 1. GSH levels in erythrocytes, brain, liver and kidney. Values are means, with standard deviations represented by vertical bars. ${ }^{*}$ Mean values were significantly different from the control. † Mean values were significantly different from the TCA-exposed rats $(P<0.05$, one-way ANOVA). $\square$, Control; 圈, TCA; 图, TCA + Sideritis caesarea.

water-bath for $15 \mathrm{~min}$ and cooled to room temperature. The optical density was measured at 532 and $600 \mathrm{~nm}$ in a spectrophotometer (Novaspec II Pharmacia-Biotech, Biochrom Limited, Cambridge, UK) for the MDA concentration in erythrocytes, and optical density was measured at $532 \mathrm{~nm}$ for the MDA concentration in tissues.

The GSH concentration in erythrocytes and tissues was measured using the method described by Beutler et al. ${ }^{(18)}$. Briefly, $0.2 \mathrm{ml}$ of fresh erythrocyte pellets or supernatant was added to $1.8 \mathrm{ml}$ of distilled water. Then, $3 \mathrm{ml}$ of the precipitating solution $(1.67 \mathrm{~g}$ metaphosphoric acid, $0.2 \mathrm{~g}$ EDTA and $30 \mathrm{~g} \mathrm{NaCl}$ in $100 \mathrm{ml}$ of distilled water) were mixed with haemolysate. The mixture was allowed to stand for approximately $5 \mathrm{~min}$ and then filtered (Whatman no. 42). The filtrate $(2 \mathrm{ml})$ was taken and added into another tube, and then $8 \mathrm{ml}$ of the phosphate solution $\left(0.3 \mathrm{M}\right.$-disodium phosphate) and $1 \mathrm{ml}$ of $5,5^{\prime}$-dithiobis(2-nitrobenzoic acid) were added. A blank was prepared with $8 \mathrm{ml}$ of the phosphate solution, $2 \mathrm{ml}$ of diluted precipitating solution (three parts to two parts of distilled water) and $1 \mathrm{ml}$ of the 5,5'-dithiobis-(2-nitrobenzoic acid) reagent. A standard solution of glutathione was prepared $(40 \mathrm{mg} / 100 \mathrm{ml})$. The optical density was measured at $412 \mathrm{~nm}$ in the spectrophotometer.

CAT (EC 1.11.1.6) activity was determined using the method described by Beutler ${ }^{(19)}$. Briefly, $1 \mathrm{~m}$-Tris-HCl, 5 mm-EDTA $(\mathrm{pH} 8), 10 \mathrm{~mm}-\mathrm{H}_{2} \mathrm{O}_{2}$ and water were mixed, and the rate of $\mathrm{H}_{2} \mathrm{O}_{2}$ consumption at $240 \mathrm{~nm}$ and $37^{\circ} \mathrm{C}$ was used for the quantitative determination of CAT activity ( $20 \mu \mathrm{l}$ of $95 \%$ ethanol were added in $1 \mathrm{ml}$ of the haemolysate to break down the complex of CAT and $\mathrm{H}_{2} \mathrm{O}_{2}$ ). GST (EC 2.5.1.18) activity was assayed at $25^{\circ} \mathrm{C}$ spectrophotometrically by following the conjugation of glutathione with 1-chloro-2,4-dinitrobenzene at $340 \mathrm{~nm}$, as described by Mannervik \& Guthenberg ${ }^{(20)}$. GR (EC 1.6.4.2) activity was assayed at $37^{\circ} \mathrm{C}$ and $340 \mathrm{~nm}$ by following the oxidation of NADPH by $G_{S S G}^{(19)}$. SOD (EC 1.15.1.1) activity was measured at $505 \mathrm{~nm}$ and $37^{\circ} \mathrm{C}$ and calculated using the inhibition percentage of formazan dye ${ }^{(21)}$.

\section{Analysis of the data}

All data were expressed as means and standard deviations. The statistical analyses were performed using Minitab 13 for windows (State College, PA, USA). Means and standard deviations were calculated according to the standard methods for all parameters. A one-way ANOVA statistical test was used to determine the differences between means of the treatment and control groups, accepting the significance level at $P \leq 0 \cdot 05$.

\section{Results}

Following the exposure of the experimental groups, the effects of the plant's infusion antioxidative role on TCA were evaluated by examining antioxidant defence system

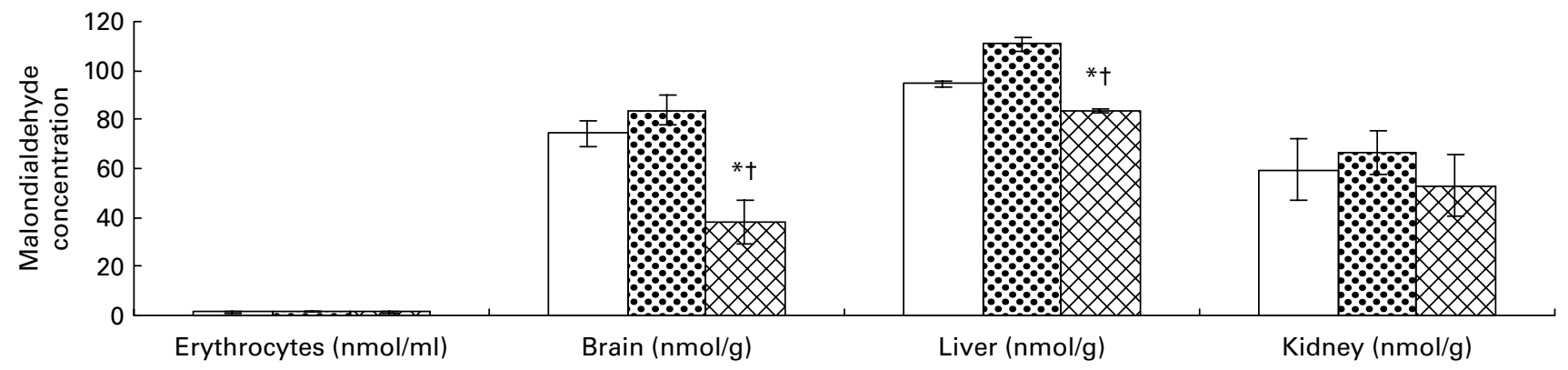

Fig. 2. Malondialdehyde levels in erythrocytes, brain, liver and kidney. Values are means, with standard deviations represented by vertical bars. ${ }^{\star}$ Mean values were significantly different from the control. † Mean values were significantly different from the TCA-exposed rats $(P<0 \cdot 05$, one-way ANOVA). $\square$, Control; 8 , TCA; , TCA + Sideritis caesarea. 


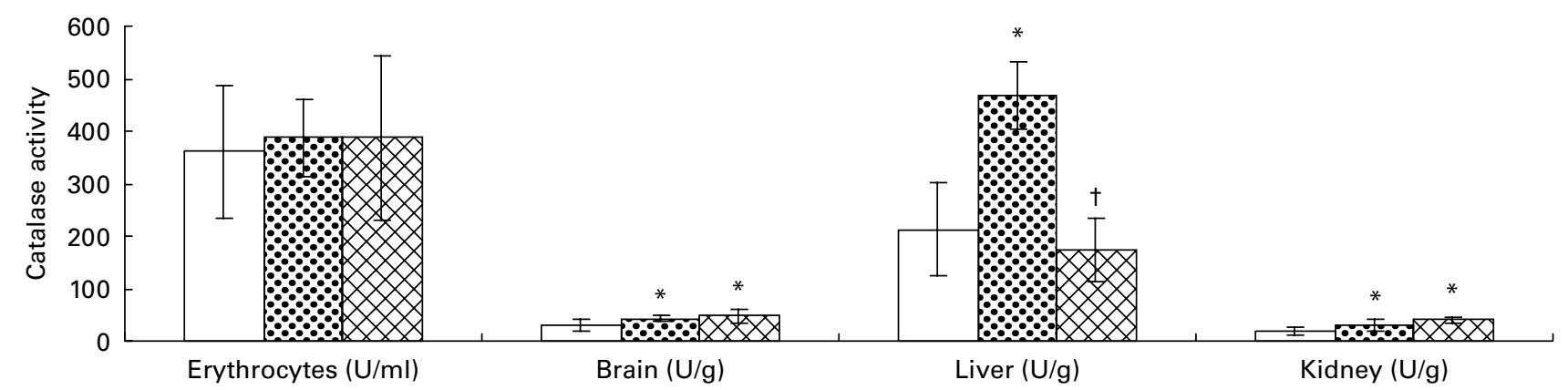

Fig. 3. Catalase activity in erythrocytes, brain, liver and kidney. Values are means, with standard deviations represented by vertical bars. ${ }^{*}$ Mean values were sig-

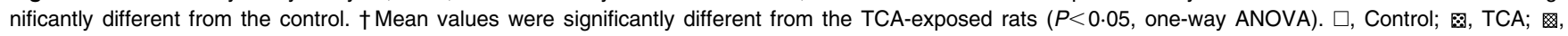
TCA + Sideritis caesarea.

constituents such as GSH, CAT, GST, GR and SOD, and MDA contents in the erythrocytes, and liver, brain and kidney tissue samples obtained from the control and treated rats. The results of the experiment showed that the treatment of rats with TCA and TCA + plant infusion caused changes in the MDA concentration and antioxidative defence systems in the erythrocytes, liver, brain and kidneys in comparison with the control rats (Figs. 1-6). According to the results, MDA content (Fig. 2) increased significantly in tissues of rats exposed to TCA, whereas it decreased in group $\mathrm{C}$ rats. With regard to antioxidant enzyme activities, SOD (Fig. 5) and CAT (Fig. 3) increased significantly in the brain, liver and kidneys of group B rats, while they decreased significantly in group $\mathrm{C}$ rats as compared with the TCA-exposed rats. The GSH (Fig. 1) level and the ancillary enzyme GR (Fig. 4) activity increased significantly in the brain and liver of group $\mathrm{C}$ rats as compared with the control and TCA-exposed rats. On the other hand, the activity of the drug-metabolising enzyme GST (Fig. 6) decreased significantly in the brain and liver of group $\mathrm{C}$ rats, while a significant increase was observed in erythrocytes.

\section{Discussion}

Overexposure to oxidative stress caused by environmental pollutants is thought to increase the risk of cancer. Hence, efforts are needed to provide effective protection against damaging agents. Experimental studies have implicated the influence of a number of plant chemicals in this regard. The first aim of the present study was to investigate whether SC could prevent oxidative stress caused by TCA.

As shown in Figs. 1-6, the results of the present study demonstrated that a beverage made from the plant SC could have antioxidative capacity against TCA toxicity in rats. This was obvious from our observation that, as a result of the plant's infusion with an additional treatment in vivo, the concentration of MDA in the erythrocytes, liver, brain and kidneys differed from that of the TCAexposed group. According to the obtained results, MDA concentrations increased in the erythrocytes, liver and kidneys of rats treated with TCA, whereas they decreased in the SC infusion-supplemented groups. The reasons for such an effect of TCA and the SC beverage are not understood yet. But the increased MDA content might have resulted from an increase in reactive oxygen species as a result of stress in rats with TCA intoxication. Also, it is known that an elevation of MDA results after some xenobiotics are consumed and followed often by superoxide overproduction, after which dismutation produces singlet oxygen and $\mathrm{H}_{2} \mathrm{O}_{2}$, easily converted later into the reactive $\cdot \mathrm{OH}$. Both singlet oxygen and the $\mathrm{OH}$ radical have a high potential to initiate free-radical chain reactions of lipid peroxidation. Also, it is known that $\cdot \mathrm{OH}$ can initiate lipid

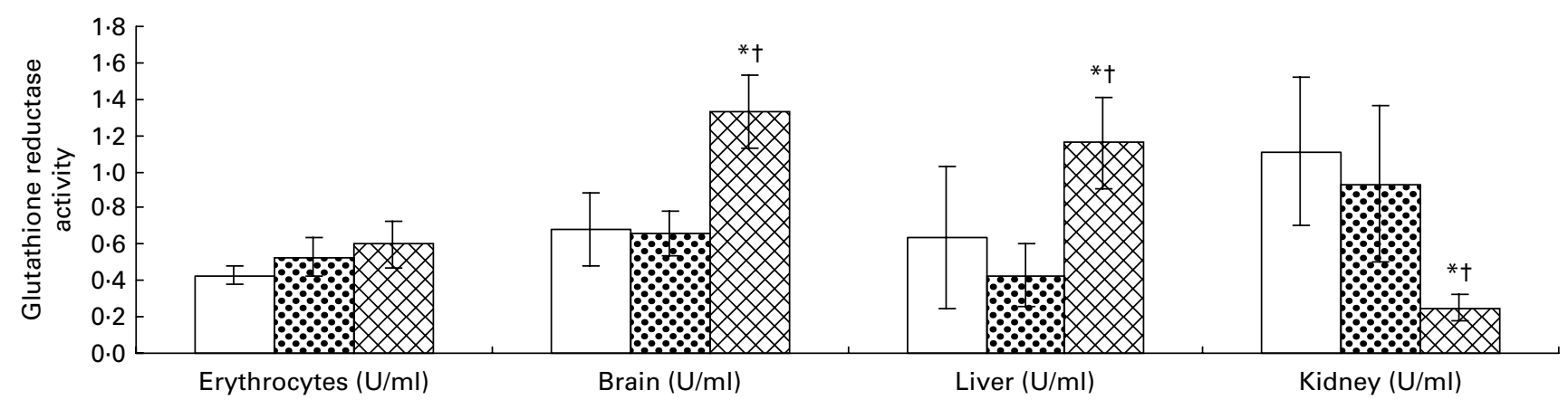

Fig. 4. Glutathione reductase activity in erythrocytes, brain, liver and kidney. Values are means, with standard deviations represented by vertical bars. ${ }^{*}$ Mean values were significantly different from the control. † Mean values were significantly different from the TCA-exposed rats $(P<0 \cdot 05$, one-way ANOVA). $\square$, Control; 图, TCA; 图, TCA + Sideritis caesarea. 


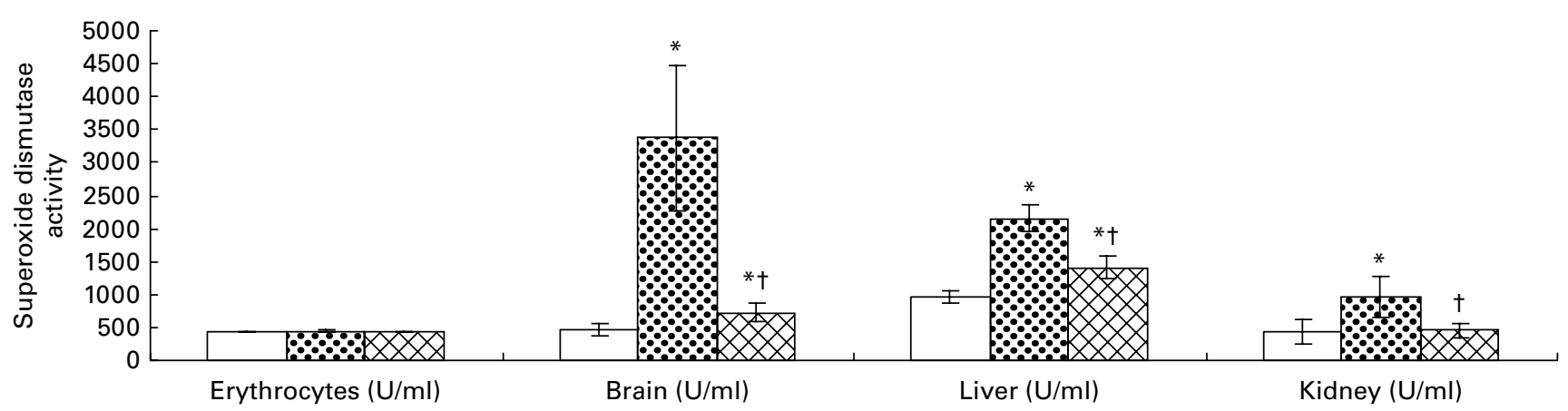

Fig. 5. Superoxide dismutase activity in erythrocytes, brain, liver and kidney. Values are means, with standard deviations represented by vertical bars. ${ }^{*}$ Mean values were significantly different from the control. † Mean values were significantly different from the TCA-exposed rats $(P<0.05$, one-way ANOVA). $\square$, Control; 圈, TCA; $⿴ 囗 \mathbf{x}, \mathrm{TCA}+$ Sideritis caesarea.

peroxidation in tissues ${ }^{(13)}$, and MDA is a major oxidation product of peroxidised PUFA and increased MDA content is an important indicator of lipid peroxidation ${ }^{(22)}$.

The results of the present study also showed that antioxidant enzyme activities such as SOD and CAT increased significantly in the brain, liver and kidneys of the TCAexposed group, whereas they almost remained or decreased in the SC infusion-supplemented groups as compared with the control and TCA-treated rats. Meanwhile, the ancillary enzyme GR activity and GSH levels in the erythrocytes, liver and kidney did not change in all treatment groups but increased in group C. On the other hand, while the activity of the drug-metabolising enzyme GST decreased significantly in the liver, brain and kidneys of the TCA + SC group compared with both the TCAexposed and control rats, it did not change in the tissues of the TCA + SC group as compared with that of the control group. The increased activities of SOD and CAT are known to serve as protective responses to eliminate reactive free radicals ${ }^{(23)}$. The decreased activity of GST may lead to decreased protection against oxidants ${ }^{(24)}$. Doyotte et $a l^{(25)}$ pointed out that a decreased GST response might accompany a first exposure to pollutants, which can be followed by an induction of antioxidative systems. Thus, the existence of an inducible antioxidant system may reflect an adaptation of organisms. Also, the reasons for such an effect of the plant's infusion may be due to strong antioxidant and metal-chelating properties exhibited by flavonoids present in the plants, thereby protecting cells and tissues against free oxygen radicals. So far, no study examining the preventive role of SC infusion in vivo has been made on rat antioxidant defence systems and MDA content using a drinking water infusion. Therefore, we had no opportunity to compare the present results with previous ones. In addition, because of a high variability in analysing the serum enzyme-chemical interaction in vitro and in vivo, inconsistent factors such as treatment time and manner, the settings of the studies and species tissue differences, etc., it is difficult to compare the present data with different studies on chemopreventive properties. However, Tunalier et al. ${ }^{(26)}$ investigated that petroleum diethyl ether extracts of the Sideritis sp. have antioxidative properties in vitro. Also, antioxidant activities of Sideritis raeseri and Sideritis javalambrensis have been reported $^{(27,28)}$. Despite treatment time and manner and the different settings of the studies, the results of the aforementioned studies are in agreement with the present results.

In conclusion, the observations in the present study led us to conclude that while the administration of TCA increases MDA concentration and causes fluctuation in antioxidative systems, a water extract of the plant SC may possess an antioxidant role against oxidative stress caused by TCA in rats. On the other hand, the findings of the present study suggest that further experiments

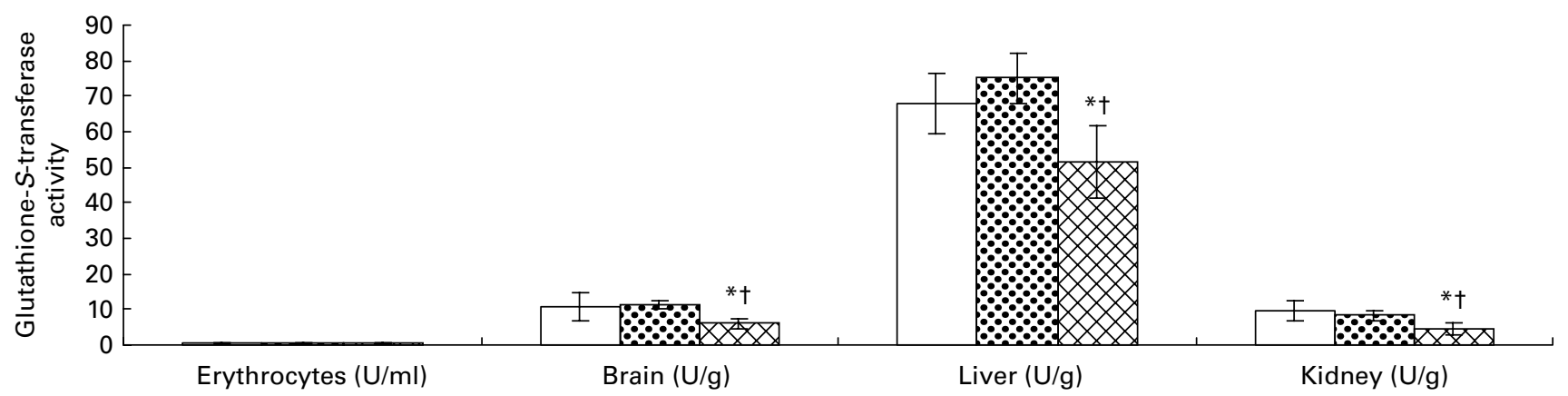

Fig. 6. Glutathione-S-transferase activity in erythrocytes, brain, liver and kidney. Values are means, with standard deviations represented by vertical bars. ${ }^{*}$ Mean values were significantly different from the control. † Mean values were significantly different from the TCA-exposed rats $(P<0.05$, one-way ANOVA). $\square$, Control; 图, TCA; 図, TCA + Sideritis caesarea. 
should be performed to elicit what is responsible for decreasing or increasing the level of the antioxidant defence systems and MDA concentration.

\section{Acknowledgements}

None of the authors had a commercial interest, financial interest and/or other relationships with manufacturers of pharmaceuticals, laboratory supplies and/or medical devices or with commercial providers of medically related services. The authors are grateful to the University of Yuzuncu Yil Grant Commission for providing financial assistance during the tenure of research. I. C. was the main moderator of the study. I. C. and M. S. K. performed the biochemical investigation and treatment in the present study.

\section{References}

1. Schulman RN \& Heather SO (2003) Plants provide new drug leads: the natural medicinal properties of plants could save millions in drug development costs. Prepared Foods 172, $13-18$.

2. Gurbuz I, Ozkan MA, Yesilada E, et al. (2005) Anti-ulcerogenic activity of some plants used in folk medicine of Pinarbasi (Kayseri, Turkey). J Ethnopharmacol 101, 313-318.

3. Alcaraz MJ \& Tordea M (1988) Studies on the gastric antiulcer activity of hypolaetin-8-glycoside. Phytother Res 2 , 85-88.

4. Barberan FAT, Manez S \& Villar A (1987) Identification of antiinflammatory agents from Sideritis species growing in Spain. J Nat Prod 50, 313.

5. Celik I \& Isik I (2008) Determination of chemopreventive role of Foeniculum vulgare and Salvia officinalis infusion on trichloroacetic acid-induced increased serum marker enzymes lipid peroxidation and antioxidative defense systems in rats. Nat Prod Res 22, 66-75.

6. Gülçin I (2006) Antioxidant activity of caffeic acid (3,4dihydroxycinnamic acid). Toxicology 217, 213-220.

7. Gülçin I (2006) Antioxidant and antiradical activities of L-carnitine. Life Sci 78, 803-811.

8. Perchellet JP \& Perchellet EM (1989) Antioxidant and multistage carcinogenesis in mouse skin. Free Radic Biol 7, $377-408$

9. Ak T \& Gülçin I (2008) Antioxidant and radical scavenging properties of curcumin. Chem Biol Interact 174, 27-37.

10. Gülçin I, Tel AZ \& Kirecci E (2008) Antioxidant, antimicrobial, antifungal, and antiradical activities of Cyclotrichium niveum (boiss.) manden and scheng. Int J Food Propert 11, 450-471.

11. Gülçin I, Mshvildadze V, Gepdiremen A, et al. (2006) Screening of antiradical and antioxidant activity of monodesmosides and crude extract from Leontice smirnowii tuber. Phytomedicine 13, 343-351.

12. Vendemiale G, Grattagliano I \& Altomare E (1999) An update on the role of free radicals and antioxidant defense in human disease. Int J Clin Lab Res 29, 49-55.

13. Halliwell B (1996) Oxidative stress, nutrition and health. Experimental strategies for optimization of nutritional antioxidant intake in humans. Free Radic Res 25, 57-74.

14. Saha P \& Das S (2003) Regulation of hazardous exposure by protective exposure modulation of phase II detoxification and lipid peroxidation by Camellia sinensis and Swertia chirata. Teratog Carcinog Mutatgen Suppl. 1, 313-322.

15. WMA (2000) World Medical Association Declaration of Helsinki, 52nd WMA General Assembly, Edinburgh, Scotland.

16. ACGIH (1991) Documentation of the Threshold Limits Values and Biological Exposure Indices, 6th ed. Cincinnati, $\mathrm{OH}$ : American Conference of Governmental Industrial Hygienists.

17. Jain SK, McVie R, Duett J, et al. (1989) Erythrocyte membrane lipid peroxidation and glycolylated hemoglobin in diabetes. Diabetes 38, 1539-1543.

18. Beutler E, Dubon OB \& Kelly M (1963) Improved method for the determination of blood glutathione. J Lab Clin Med 61, 882-888.

19. Beutler E (1984) Red Cell Metabolism: A Manual of Biochemical Methods, 3rd ed. New York: Grune \& Startton pp. 105-106.

20. Mannervik B \& Guthenberg C (1981) Glutathione $S$-transferase (human placenta). Method Enzymol 77, 231-235.

21. McCord JM \& Fridovich I (1969) Superoxide dismutase, an enzymatic function for erythrocuprein (hemocuprein). $J$ Biol Chem 244, 6049-6053.

22. Freeman BA \& Crapo JD (1981) Hyperoxia increases oxygen radical production in rat lung and lung mitochondria. $J$ Biol Chem 256, 10986-10992.

23. Cheung CCC, Zheng GJ, Li AMY, et al. (2001) Relationship between tissue concentrations of polycyclic aromatic hydrocarbons and antioxidative responses of marine mussels Perna viridis. Aquat Toxicol 52, 189-203.

24. Amstad P, Peskin A, Shah AG, et al. (1991) The balance between $\mathrm{Cu}, \mathrm{Zn}$-superoxide dismutase and catalase affects the sensitivity of mouse epidermal cells to oxidative stress. Biochemistry 30, 9305-9313.

25. Doyotte A, Cossu C, Jacquin MC, et al. (1997) Antioxidant enzymes, glutathione and lipid peroxidation as relevant biomarkers of experimental or field exposure in the gills and the digestive gland of the freshwater bivalve, Unio tumidus. Aquat Toxicol 39, 93-110.

26. Tunalier Z, Kosar M, Ozturk N, et al. (2004) Antioxidant properties and phenolic composition of Sideritis species. Chem Nat Compd 40, 206-210.

27. Gabrieli CN, Kefalas PG \& Kokkalou EL (2005) Antioxidant activity of flavonoids from Sideritis raeseri. J Ethnopharmacol 96, 423-428.

28. Rios JL, Manez S, Paya M, et al. (1992) Antioxidant activity of flavonoids from Sideritis javalambrensis. Phytochemistry 31 , 1947-1950. 